\title{
UPPER GASTROINTESTINAL BLEEDING AFTER CARDIAC SURGERY. A CASE REPORT
}

A. Font Gual1, A. Parera Ruiz1, J. Miralles Bagan², V. Cegarra Sanmartín1, J. Galán Serrano1, M. V. Moral García1

${ }^{1}$ Hospital de la Santa Creu i Sant Pau - Barcelona (Spain), 2Sant Antoni Maria Claret 167 - Barcelona (Spain)

\section{Bacground and Goal of Study}

Upper gastrointestinal bleeding is a rare complication that can be observed after cardiac surgery and intraoperative transesophageal echocardiography, but may have serious consequences.

\section{Case report}

73-year old female, scheduled for aortic valve replacement with bioprosthesis due to valve stenosis. Among her pathological background, only hypertension, diabetes mellitus and dislypidemia to be mentioned. Surgery took place without further complication. Monitoring was performed by means of transesophageal echocardiography. After surgery, the patient was delivered intubated to the ICU. She remained stable hemodynamically during the first hours with low doses of noradrenaline. Arterial blood sample, ECG, and chest ray were normal, therefore weaning was decided. During the process of weaningwe observed important hematemesis. An important clot was seen in gastric fundus as well as traces of fresh blood with no further injuries. The injury was sclerosed with adrenaline without direct vision. Due to persisting bleeding and hemodinamic instability, the clot could be removed the following day and an important subcardial bleeding injury was clipped, achieving a bleeding stop. The patient remained stable, without further bleeding during the following hours and could be satisfactorily extubated. One week later the patient was discharged with no further incidence.

\section{Results and Discussion}

The location of the gastric lesion led us to suspect a lesion secondary to intraoperative TEE as the main cause, given the absence of digestive pathological background. There have been several reports about TEE associated gastroesophageal injury, with an incidence range between 0,03 and $1 \%$. Although TEE is a useful diagnostic tool during open heart surgery, considered relatively safe and noninvasive, the TEE probe is associated with complications such as gastroesophageal injury. Repeated trauma, mucosal hypoperfusion, heparin use and digestive background are the major risk factors. A careful use of the probe, the insert before heparinization, keeping hemodynamic stability and considering EGD before TEE in those patients with a background of digestive symptoms could help to reduce the incidence of this rare but potentially serious complication.
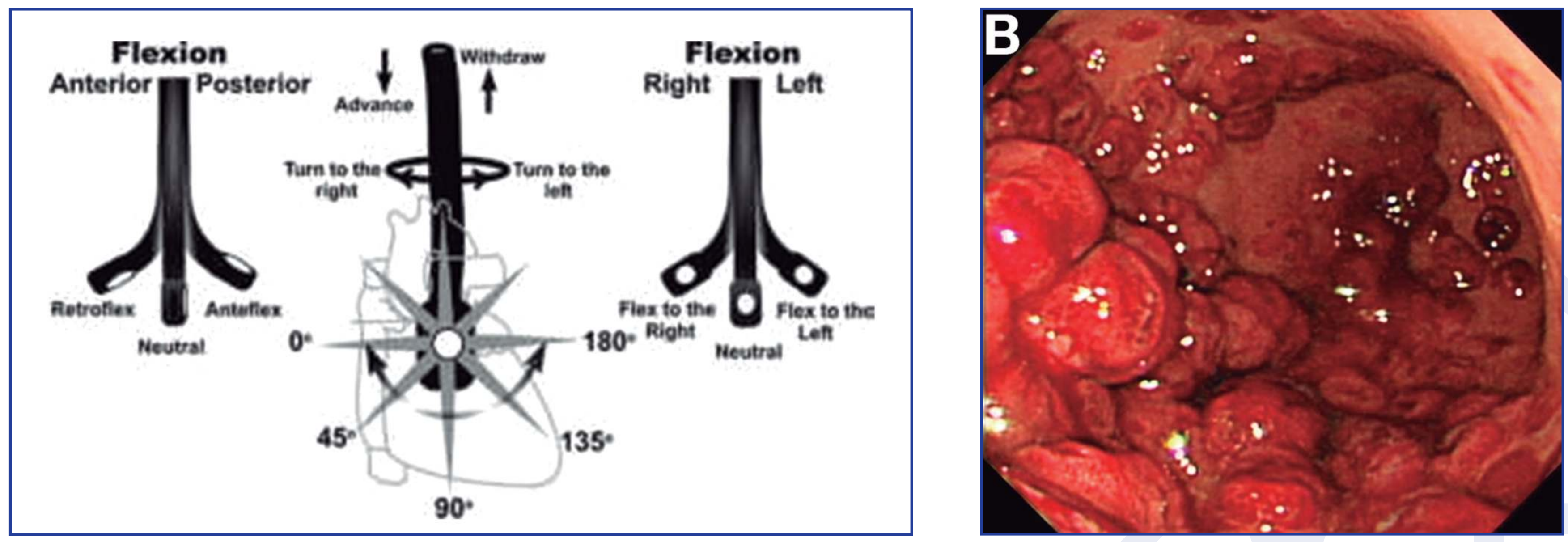

Although being a safe technique, we believe that it is essential to take measures that reduce the incidence of gastrointestinal bleeding, due to its potentially fatal consequences. 